Submitted to J. Electrochem. Soc., December, 2001

\title{
Characterization of the SEI on a carbon film electrode by combined EQCM and Spectroscopic Ellipsometry
}

\author{
Kyungjung Kwon, Fanping Kong, Frank McLarnon and \\ James W. Evans*
}

Dept. of Materials Science and Engineering,

University of California

and

Environmental Energy Technologies Division

Lawrence Berkeley National Laboratory

Berkeley, Ca. 94720

* To whom correspondence should be addressed.

Fax: 510-642-9164

e-mail: evans@socrates.berkeley.edu 


\begin{abstract}
The electrochemical quartz crystal microbalance (EQCM) and cyclic voltammetry have been applied simultaneously to characterize electron-beam deposited carbon film electrodes in $\mathrm{LiClO}_{4}$ or $\mathrm{LiPF}_{6}$-containing mixed electrolytes of ethylene carbonate (EC) and dimethyl carbonate (DMC). The structure of the carbon electrode was found to be amorphous/disordered using Raman spectroscopy. Cyclic voltammetry in $\mathrm{LiClO}_{4} / \mathrm{EC}+\mathrm{DMC}$ demonstrated features typical of $\mathrm{Li}$ intercalation/deintercalation into/from the disordered carbon electrode, and EQCM showed a corresponding mass increase/decrease. Contrary to the case of $\mathrm{LiClO}_{4} / \mathrm{EC}+\mathrm{DMC}$ electrolyte, $\mathrm{LiPF}_{6} / \mathrm{EC}+\mathrm{DMC}$ electrolyte showed no Li deintercalation out of the thin-film carbon electrode. Combined EQCM and spectroscopic ellipsometry data were compared, and the solid electrolyte interphase density after the first cycle in $\mathrm{LiClO}_{4} / \mathrm{EC}+\mathrm{DMC}$ was estimated to be $1.3 \mathrm{~g} / \mathrm{cm}^{3}$.
\end{abstract}




\section{Introduction}

Carbon electrodes, in both graphitic and amorphous forms, are used as negative electrodes in secondary Li-ion batteries. The limiting capacity of $\mathrm{LiC}_{6}$ is $372 \mathrm{mAh} / \mathrm{g}$ for graphite, but higher capacities have been observed with certain disordered carbons. When a carbon electrode in a non-aqueous electrolyte is charged for the first time from its open-circuit voltage (OCV) down to $0 \mathrm{~V}$ vs. $\mathrm{Li} / \mathrm{Li}^{+}$, $\mathrm{Li}$ ions are intercalated into the carbon structure. The potential region over which Li ions are intercalated depends on the structure of the carbon and the type of electrolyte. Along with Li intercalation, other electrochemical/chemical reactions that occur during the first few cycles are electrolyte decomposition on the carbon surface and the formation of an electrode surface layer, often referred to as the solid electrolyte interphase (SEI). The reactions involved in SEI formation are associated with so-called irreversible capacity loss. The SEI properties and chemical composition have been subjects of intense scientific interest because the SEI plays a critical role in the safety and cycle life of secondary Li-ion batteries. ${ }^{1-5}$ The current understanding of the role of the SEI is that solvated Li ions in the liquid electrolyte lose their solvation shells while penetrating the SEI and are incorporated into the carbon structure in a solvent-free form. In addition, the SEI not only dramatically slows the kinetics of electrolyte decomposition but 
also reduces active lithium consumption by forming a physical barrier between the lithiated carbon electrode and the electrolyte.

Spectroscopic ellipsometry (SE) is a surface-sensitive tool that is capable of detecting changes in the surface composition/structure at the molecular level. Even a small change in species adsorption on a surface or the formation of nanometer-scale surface film can lead to measurable changes in the ellipsometry parameters $\Delta$ and $\Psi$. However, to obtain a direct and unambiguous correlation between the detailed processes occurring on the electrode surface with the changes in the ellipsometric parameters requires precise information on the optical properties of the surface and all candidate film components.

The electrochemical quartz crystal microbalance technique is based on the piezoelectric effect of a quartz crystal placed between two electrodes that induce an alternating electric field. An advantage of EQCM is its ability to simultaneously measure the in situ mass change and charge involved in electrochemical processes. The EQCM allows us to measure in situ mass variation without disturbing the electrode and to combine it with such other electrochemical techniques as cyclic voltammetry (CV) and chronoamperometry. ${ }^{6,7}$ The basic feature of this technique is the linear relationship (a.k.a. the Sauerbrey equation) between the mass change per area on the working electrode and the resulting change in the resonance frequency. 
However, the actual frequency change measured during the electrochemical/chemical processes is the sum of contributions that arise from mass change due to chemical reactions, temperature change, viscoelastic change, and roughness effects in addition to the mass change associated with electrochemical reactions. ${ }^{8,9}$ In a situation as complicated as the above, the use of EQCM as a single tool for the analysis of the reaction products of these processes may be inadequate. Therefore, we have combined the EQCM and SE to characterize surface films formed on a carbon electrode in $\mathrm{LiClO}_{4}$ / ethylene carbonate $(\mathrm{EC})+$ dimethyl carbonate $(\mathrm{DMC})$ electrolyte. We anticipate that combining these highly sensitive and complementary techniques will result in improved understanding and better models of both $\mathrm{Li}$ intercalation/deintercalation processes and SEI formation on carbon electrodes.

\section{Experimental}

EC and DMC were purchased from Grant Chemical, and anhydrous propylene carbonate (PC) was purchased from Aldrich. The solvents were filtered with dry alumina from ICN and then dried over molecular sieves prior to use. $\mathrm{LiClO}_{4}(99.99 \%)$ from Aldrich was used as received. The electrolyte was $1 \mathrm{M} \mathrm{LiClO}_{4} / \mathrm{EC}+\mathrm{DMC}(1: 1$ vol. ratio) unless indicated otherwise. 
The $6 \mathrm{MHz}$ quartz crystal used for working-electrode substrates was purchased from International Crystal Manufacturing (ICM). To fabricate a thin-film carbon electrode, PVD (electron beam evaporation of a carbon target) was used to deposit a 100-nm thick carbon film onto one side of a smooth AT-cut quartz substrate pre-coated with two transition layers $(500-\mathrm{nm} \mathrm{Cu}$ and 10-nm Cr). The electron beam current was approximately $100 \mathrm{~mA}$ at $10 \mathrm{kV}$ with the deposition chamber at $10^{-6}$ to $10^{-5}$ torr. The surface areas of the working electrodes were $0.374 \mathrm{~cm}^{2}$. ICM applied a $1-\mu \mathrm{m}$ thick Au or Ni layer onto the other side of each quartz substrate. The quartz substrates were $12.7 \mathrm{~mm}$ in diameter and $0.25 \mathrm{~mm}$ thick. For SE measurements, a 100-nm thick carbon film was deposited onto a well-finished glass substrate with similar transition layers, and the electrode working area was about $20 \mathrm{~cm}^{2}$ to match the ellipsometric cell configuration.

A standard three-electrode cell was used in all electrochemical experiments. Lithium from Cyprus Foote Mineral Company was used for reference and counter electrodes. The galvanostatic cycling experiment employed a constant current density of $10 \mu \mathrm{A} / \mathrm{cm}^{2}$, and the CV used a scan rate of $1 \mathrm{mV} / \mathrm{s}$ for 20 cycles between $0 \mathrm{~V}$ and $2 \mathrm{~V}$ vs. $\mathrm{Li} / \mathrm{Li}^{+}$, except for the first cycle starting from the OCV. All EQCM experiments were conducted in a glove box with an inert atmosphere (argon with water and oxygen concentrations less than $5 \mathrm{ppm}$ ). The 
EQCM instrument and potentiostat were assembled in our laboratory. The system and oscillator circuit were described previously. ${ }^{10,11}$ The EQCM instrument and potentiostat were controlled by a data acquisition board (PCI-DAS1200, Computer Boards). For SE measurements, a similar three-electrode cell with two optical windows was used. Details of the cell design and the spectroscopic ellipsometer have been published. ${ }^{1}$ The cell was assembled in a glove box and transferred to the SE after a stabilization period.

Raman spectroscopy of the thin-film carbon samples was carried out with a Spectraphysics Model 127, $35 \mathrm{~mW}$ He-Ne laser (632.8nm). The Raman-scattered radiation was dispersed by a Spex 500M spectrometer equipped with a 1200 groove/mm diffraction grating. A Spectrum 1 CCD camera was positioned at the exit of the spectrometer and recorded the intensity of the dispersed radiation. A typical spectrum was obtained with a 50 second exposure time and a $100 \mathrm{~mm}$ x $2 \mathrm{~mm}$ wide entrance slit opening.

\section{Results and discussion}

$\underline{\text { Structural and electrochemical characterization of the carbon film electrode. }}$

Raman spectroscopy was used to characterize the thin-film carbon electrode, and the Raman spectra are shown in Fig.1. There is no visible dominant peak which is characteristic of graphite, diamond, glassy carbon, or any other form of 
ordered carbon but rather the superposition of all possible vibrations of various carbon structures. This is typical for a highly disordered/amorphous or microcrystalline phase. Although the origin of the spectrum is unknown, it might be attributed to vibrations of damaged carbon rings, particularly when the size of carbon macromolecules is close to the physical size of carbon grains or other features. Therefore, the Raman data indicate that the carbon film is mostly amorphous (broad peaks in the range $1300-1600 \mathrm{~cm}^{-1}$ ), but some minor changes are observed after the cycling (for example, the peak at $\sim 1500 \mathrm{~cm}^{-1}$ is enhanced in the Raman spectra recorded after CV). However, it is difficult to conclude that cycling induced a significant change in the carbon structure.

A galvanostatic cycling test, which is the most widely used technique for evaluating carbon materials, was conducted, and the results appear in Fig. 2. The potential decreased from the OCV until it reached $0 \mathrm{~V}$ vs. $\mathrm{Li} / \mathrm{Li}^{+}$during charging, and during discharging the potential returned to $2 \mathrm{~V}$, which is the usual cutoff potential. Note that there is a large irreversible charge and there are no plateaus. First, the large irreversible cathodic charge is due to SEI formation, as is also seen in the next CV (Fig. 3a). The anodic capacity in the first cycle is almost $800 \mathrm{mAh} / \mathrm{g}$, which is extraordinary for graphite materials. This value is similar to the reversible capacity of disordered carbons during the first cycle, as reported by Takami et al.. ${ }^{12}$ This large capacity drops to about half of the 
theoretical capacity for graphite at later cycles. Second, the potential gradually increases or decreases so that we cannot observe a distinct plateau which would imply staging phenomena in a graphitic structure. ${ }^{13}$ These features are expected results when the amorphous-like structure of carbon film is considered.

\section{EQCM study of the SEI.}

CV and EQCM were applied simultaneously to characterize a carbon film electrode in $1 \mathrm{M} \mathrm{LiClO}_{4} / \mathrm{EC}+\mathrm{DMC}$ electrolyte. The measured quantities (current density, $\mathrm{i}$, and resonant frequency change, $\Delta \mathrm{f}$ ) for both techniques are plotted in Fig. 3. Because the mass increase corresponds to a decrease in the resonant frequency, the negative of $\Delta \mathrm{f}$ is plotted versus potential to facilitate direct interpretation. Both the current density and frequency changes for the first cycle are larger than those for subsequent cycles. From the second cycle to the $20^{\text {th }}$ cycle (not shown from the $4^{\text {th }}$ cycle), the shapes of the CVs and electrogravimetric curves are preserved. For the first cathodic scan, an irreversible peak appears at about $0.8 \mathrm{~V}$, which is the same peak reported by Takamura et al.. ${ }^{14}$ Although Li intercalation (which is assumed to constitute the major part of the cathodic current after the first cycle) prevails as the potential approaches $0 \mathrm{~V}$, Li deintercalation (which is believed to correspond to anodic current) is significant only in the potential range above $0.5 \mathrm{~V}$. This $\mathrm{Li}$ 
intercalation/deintercalation behavior has been reported in papers dealing with disordered carbon. ${ }^{12,15}$ The frequency responses in Fig. 3b coincide qualitatively with the current behavior, that is, the frequency decreases with increasing cathodic current and increases with increasing anodic current, thereby representing Li intercalation/deintercalation.

Although this frequency response coincides qualitatively with the current behavior, there exists a quantitative discrepancy between them. Figure 4 shows measured frequency and charge changes oscillating as a function of time during $\mathrm{CV}$. Although the charge stabilizes in later cycles, becoming almost reversible, the frequency continues to decrease (with a decreasing amplitude of oscillation). This discrepancy is due to the viscosity-induced frequency change as reported previously. ${ }^{16}$ As detailed in that previous report, measurements on electrolytes in contact with the EQCM crystals revealed an increasing viscosity. Subsequent experiments have shown the increase to be due to contact of the electrolyte with the quartz. The gradual change of frequency observed in the present investigation results, not from changes on the electrode, but from changes in electrolyte viscosity and is a function of time. Therefore, the viscosity-induced frequency, which is estimated from the frequency change at $\mathrm{OCV}$, will be used as a base-line frequency to separate the frequency change originating from the change of the electrode from the total measured frequency change. 
This approach is shown in Fig. 5 where the first frequency decrease from $\mathrm{OCV}$ to $\sim 1.5 \mathrm{~V}$ deviates little from the frequency change at $\mathrm{OCV}$, because a very small current flows in this potential range. When the current becomes substantial below $1.5 \mathrm{~V}$, the frequency decreases sharply, indicating film formation on the carbon electrode.

The corrected frequency change (adjusted by the frequency change at OCV) in Fig. 4 shows that the frequency stabilizes after about the $16^{\text {th }}$ cycle (corresponding to about 60000 seconds) as does the charge. Although there is no guarantee that the frequency change at OCV exactly reflects the viscosity-induced frequency change during the course of $\mathrm{CV}$, the corrected data appear convincing enough to argue that the frequency does not continue to decrease as it looks at later cycles.

$\underline{\text { SE study of the SEI. }}$

$\mathrm{CV}$ and SE were applied simultaneously to characterize a carbon film electrode in $1 \mathrm{M} \mathrm{LiClO}_{4} / \mathrm{EC}+\mathrm{DMC}$ electrolyte. The ellipsometry parameters $\Delta$ and $\Psi$ were measured at a wavelength of $500 \mathrm{~nm}$ during the first three cycles. The CV was similar to that in Fig. 3a, and the measured ellipsometry parameters are plotted vs. time in Fig. 6 . The SE data show that the changes in $\Delta$ and $\Psi$ during the first cycle are different from those during the second and third 
cycles. The repetitive character of SE parameter changes during the second and third cycles can be attributed to reversible Li intercalation/deintercalation, and the changes during the first cycle are due to irreversible changes at the carbon surface as well as Li intercalation/deintercalation ${ }^{1}$.

To obtain more quantitative information on the SEI, ellipsometric spectra were recorded over the spectral range $410-560 \mathrm{~nm}$, before and after the first cycle (Fig. 7). To extract physical properties of the SEI from these SE data, two optical models were developed and evaluated using simulation calculations. The first model considered the carbon film to be compact and homogeneous, and the second model took the porosity and the surface roughness of the carbon film into account by using an effective medium approximation. Surface roughness data (measured using atomic force microscopy) were used to select initial trial values and thereby improve the accuracy and efficiency of data fitting. [The AFM images showed a roughness of the order of $2 \mathrm{~nm}$ on a length scale of approximately $100 \mathrm{~nm}$ and the roughness was unchanged after exposure to electrolyte.] The second model showed better fitting results and indicated that the SEI was about $60 \mathrm{~nm}$ thick after the first scan. Deconvolution of experimental data also pointed out that the SEI refractive index fell in the range 1.41 to 1.52 and its extinction coefficient was close to zero in the visible spectrum. These optical data suggest that the SEI layer is optically compact 
(compared to common low-loss polymers) and has almost no electronic conductivity.

Estimation of the SEI density.

Before the EQCM and SE data are compared to estimate the SEI density, an apparent discrepancy between them should be addressed. Although SE shows that the SEI was almost completely formed during the first cycle, the EQCM frequency changes persisted in subsequent cycles. In particular, the frequency changes over the potential range from OCV to about $1.5 \mathrm{~V}$ (where negligible currents flow) are the most conspicuous difference. The SE parameters show slight changes over this potential range and rise at about $1.3 \mathrm{~V}$ (see Fig. 6). As discussed earlier, the origin of the frequency change from OCV to $1.5 \mathrm{~V}$ is due, not to changes on the electrode, but to changes in electrolyte viscosity. Therefore, the frequency decrease over the range $(\mathrm{OCV}$ to $1.5 \mathrm{~V})$ is not considered to signal actual SEI formation. Accordingly, the base-line frequency correction was used for density estimation.

Because the mass increase during the first cathodic scan includes contributions from both SEI formation and $\mathrm{Li}$ intercalation, the Li intercalation portion should be excluded in determining the SEI mass. To a first approximation, the mass decrease during the first anodic scan can be used to 
evaluate the mass increase during the first cathodic scan due to $\mathrm{Li}$ intercalation on the assumption that Li intercalation is reversible. This analysis gives an SEI mass of about $8 \mu \mathrm{g} / \mathrm{cm}^{2}$. Therefore, the density of SEI can be estimated to be about $1.3 \mathrm{~g} / \mathrm{cm}^{3}$ using the SEI thickness of about $60 \mathrm{~nm}$ as determined by SE. The available density data (Table 1) of species that are thought by several research groups to exist in the SEI show that inorganic species have a greater density than polymeric species. The estimated density of $1.3 \mathrm{~g} / \mathrm{cm}^{3}$ lies between those of inorganic and polymeric species. These results therefore support the current understanding that the SEI layer is a composite of organic and inorganic species. $^{2-6,17}$

\section{EQCM and SE study of the SEI growth.}

The SEI thickness during CV was measured with SE and plotted in Fig. 8. The SE was carried out after the first, third, $6^{\text {th }}, 10^{\text {th }}$, and $20^{\text {th }}$ cycles, respectively. The SEI appears to be almost fully developed by the $10^{\text {th }}$ cycle, i.e. the thickness increase is negligible between the $10^{\text {th }}$ to $20^{\text {th }}$ cycle. For comparison, the corrected frequency change shown in Fig. 4 was inserted in Fig.

8. The corrected frequency data show the same trend as the SEI thickness at later cycles. That is, the resonant frequency does not change much after the $10^{\text {th }}$ cycle except for the small amplitude oscillation due to $\mathrm{Li}$ 
intercalation/deintercalation.

\section{Summary and Conclusions}

The EQCM and SE techniques were used to study electron-beam deposited carbon films as model electrodes in laboratory Li-ion cells. The structure of the carbon electrode was characterized using Raman spectroscopy and determined to be disordered/amorphous. The behavior of the carbon electrode in $\mathrm{LiClO}_{4}$ / EC+DMC electrolytes was examined by combining CV with EQCM. CVs in $\mathrm{LiClO}_{4} / \mathrm{EC}+\mathrm{DMC}$ demonstrated typical $\mathrm{Li}$ intercalation/deintercalation into/from disordered carbon and EQCM showed the corresponding mass changes. The SEI density after the first cycle in $\mathrm{LiClO}_{4} / \mathrm{EC}+\mathrm{DMC}$ was estimated to be $1.3 \mathrm{~g} / \mathrm{cm}^{3}$ by combining the EQCM and SE data. 


\section{Acknowledgment}

This work was supported by the Assistant Secretary for Energy Efficiency and Renewable Energy, Office of Transportation Technologies, Office of Advanced Automotive Technologies of the U.S. Department of Energy under contract no. DE-AC03-76SF00098. The help of Dr. Robert Kostecki in collecting and interpreting the Raman spectra is gratefully acknowledged. 


\section{References}

1. F. Kong, J. Kim, X. Song, M. Inaba, K. Kinoshita, and F. McLarnon,

Electrochem. Solid-State Lett., 1, 39 (1998).

2. R. Yazami, Electrochim. Acta, 45, 87 (1999).

3. E. Peled, D. Golodnitsky, C. Menachem, and D. Bar-Tow, J. Electrochem.

Soc., 145, 3482 (1998).

4. D. Aurbach, B. Markovsky, A. Shechter, Y. Ein-Eli, and H. Cohen, J.

Electrochem. Soc., 143, 3809 (1996).

5. A. Naji, J. Ghanbaja, B. Humbert, P. Willmann, and D. Billaud, J. Power

Sources, 63, 33 (1996).

6. D. Aurbach and A. Zaban, J. Electroanal. Chem., 393, 43 (1995).

7. T. Uchiyama, M. Nishizawa, T. Itoh, and I. Uchida, J. Electrochem. Soc., 147, 2057 (2000).

8. D. A. Buttry, in Electroanalytical Chemistry; A. J. Bard, Editors. Vol. 17, p.

1, Marcel Dekker, NY (1991).

9. D. A. Buttry and M. D. Ward, Chem. Rev., 92, 1355 (1992).

10. H. Yang and J. Kwak, J. Phys. Chem. B, 101, 774 (1997).

11. H. Yang, K. Kwon, T. M. Devine, and J. W. Evans, J. Electrochem. Soc., 147, 4399 (2000). 
12. N. Takami, A. Satoh, T. Ohsaki, and M. Kanda, J. Electrochem. Soc., 145, 478 (1998).

13. J. R. Dahn, R. Fong, and M. J. Spoon, Phys. Rev. B, 42, 6424 (1990).

14. T. Takamura, H. Awano, R. Takasu, K. Sumiya, and K. Sekine, $J$.

Electroanal. Chem., 455, 223 (1998).

15. Z. Ogumi and M. Inaba, Bull. Chem. Soc. Jpn., 71, 521 (1998).

16. K. Kwon and J. W. Evans, Electrochem. Solid-State Lett., 5, A59 (2002)

17. E. Endo, M. Ata, K. Tanaka, and K. Sekai, J. Electrochem. Soc., 145, 3757 (1998). 
Table 1. Densities of possible passivation films and electrodeposited species in $\mathrm{LiClO}_{4} / \mathrm{EC}+\mathrm{DMC}$.

\begin{tabular}{|c|c|}
\hline Deposited species & Density $\left(\mathrm{g} / \mathrm{cm}^{3}\right)$ \\
\hline Li metal & 0.53 \\
\hline Polyolefins & $\sim 0.9$ \\
\hline Poly(ethylene oxide) & $\begin{array}{c}1.13 \text { (amorphous) } \\
1.24 \text { (crystalline) }\end{array}$ \\
\hline $\mathrm{LiOH}$ & 1.45 \\
\hline $\mathrm{LiOH} \cdot \mathrm{H}_{2} \mathrm{O}$ & 1.51 \\
\hline $\mathrm{Li}_{2} \mathrm{O}$ & 2.01 \\
\hline $\mathrm{LiCl}$ & 2.07 \\
\hline $\mathrm{Li}_{2} \mathrm{CO}_{3}$ & 2.11 \\
\hline $\mathrm{Li}_{2} \mathrm{O}_{2}$ & 2.31 \\
\hline
\end{tabular}




\section{List of figure captions}

Figure 1. Raman spectra of the carbon film electrode before (as-received) and after $\mathrm{CV}$ in $1 \mathrm{M} \mathrm{LiClO}_{4} / \mathrm{EC}+\mathrm{DMC}($ scan rate $=1 \mathrm{mV} / \mathrm{s})$.

Figure 2. Galvanostatic cycle test during the first 3 cycles at $10 \mu \mathrm{A} / \mathrm{cm}^{2}$ in $1 \mathrm{M}$ $\mathrm{LiClO}_{4} / \mathrm{EC}+\mathrm{DMC}$.

Figure 3. (a) CV and (b) resonant frequency change of the carbon electrode during the first 3 cycles in $1 \mathrm{M} \mathrm{LiClO}_{4} / \mathrm{EC}+\mathrm{DMC}($ scan rate $=1 \mathrm{mV} / \mathrm{s})$.

Figure 4. Measured and corrected resonant frequency changes, and charge change of the carbon electrode with time during CV for the first 20 cycles in 1 $\mathrm{M} \mathrm{LiClO}_{4} / \mathrm{EC}+\mathrm{DMC}$.

Figure 5. Resonant frequency change of the carbon electrode with time during $\mathrm{CV}$ for the first 3 cycles and at $\mathrm{OCV}$ in $1 \mathrm{M} \mathrm{LiClO}_{4} / \mathrm{EC}+\mathrm{DMC}$.

Figure 6. Ellipsometric parameters measured at a wavelength of $500 \mathrm{~nm}$ during $\mathrm{CV}$ for the first 3 cycles in $1 \mathrm{M} \mathrm{LiClO}_{4} / \mathrm{EC}+\mathrm{DMC}$. 
Figure 7. Changes in optical parameters $\left(\Delta_{0} \& \Psi_{0}\right)$ before and $\left(\Delta_{1} \& \Psi_{1}\right)$ after the first cycle in $1 \mathrm{M} \mathrm{LiClO}_{4} / \mathrm{EC}+\mathrm{DMC}$.

Figure 8. Corrected resonant frequency change and SEI thickness measured by SE during $\mathrm{CV}$ in $1 \mathrm{M} \mathrm{LiClO}_{4}$ / EC+DMC. 